\title{
TTR
}

Traduction, terminologie, re?daction

\section{La traduction de la tragédie grecque en France : le tournant décisif de la période 1660-1780}

\section{Bruno Garnier}

Volume 11, numéro 1, 1er semestre 1998

Diachronie et synchronie

Diachronics and Synchronics

URI : https://id.erudit.org/iderudit/037315ar

DOI : https://doi.org/10.7202/037315ar

Aller au sommaire du numéro

\section{Éditeur(s)}

Association canadienne de traductologie

ISSN

0835-8443 (imprimé)

1708-2188 (numérique)

Découvrir la revue

Citer cet article

Garnier, B. (1998). La traduction de la tragédie grecque en France : le tournant décisif de la période 1660-1780. TTR, 11(1), 33-64.

https://doi.org/10.7202/037315ar

\section{Résumé de l'article}

La traduction de la tragédie grecque en France. Le tournant décisif de la période 1660-1780 — La traduction de la tragédie grecque en France, dont l'histoire avait commencé à la Renaissance, connaît une longue interruption au XVIIe siècle, qui correspond à la naissance et au développement de la tragédie classique française. C'est sur la période suivante, durant laquelle la traduction de la tragédie grecque a repris en France, que nous voulons mettre l'accent, parce que les façons de traduire qui ont prévalu à l'issue de ce nouvel élan ont fortement marqué l'histoire ultérieure du genre et elles ont continué de prévaloir, en ce qui concerne la tragédie grecque, jusqu'à une date récente. C'est au lendemain des vifs débats sur la manière de traduire les poètes et à la suite des progrès de l'érudition et de la philologie que s'est développée en France la Traduction analytique en prose, finit d'un intérêt nouveau pour l'Antiquité et d'un code de bonne conduite implicite des traducteurs. En réaction contre la prose glacée des traductions savantes, à l'autre extrémité du spectre interprétatif, a pris naissance la Traduction imitation, audacieusement libre, lointaine héritière des traductions de l'âge baroque. Depuis lors, le débat est polarisé entre ces deux options, alors que les conditions semblent aujourd'hui réunies pour que soit restaurée cette proximité du savant et du poète qui avait fait l'âge d'or de la traduction poétique à la Renaissance.

Tous droits réservés ( $\odot$ TTR: traduction, terminologie, rédaction — Les auteurs, Ce document est protégé par la loi sur le droit d'auteur. L’utilisation des 1998 services d’Érudit (y compris la reproduction) est assujettie à sa politique d'utilisation que vous pouvez consulter en ligne.

https://apropos.erudit.org/fr/usagers/politique-dutilisation/ 


\title{
La traduction de la tragédie grecque en France : le tournant décisif de la période 1660-1780
}

\author{
Bruno Garnier
}

Qui s'est intéressé à l'histoire de la traduction du théâtre étranger en français n'a pas manqué de relever les effets produits par la naissance et le développement de la tragédie et de la comédie classiques françaises sur les pratiques et les postulats théoriques, plus ou moins conscients, des traducteurs. Pourquoi s'étonnerait-on de constater qu'on ne traduit plus de la même façon la tragédie au temps de Robert Garnier et après Corneille et Racine?

Cependant, un regard attentif impose de mettre l'accent surtout sur les longues années qui suivent l'âge d'or de la production classique française. Appliquée à la traduction française des tragédies grecques, cette étude met en évidence les bouleversements considérables qui se font jour au cours du XVII' siècle dans les théories et dans les pratiques des traducteurs, comme une réplique tardive à la naissance de la dramaturgie classique française, et dont l'onde de choc se fait encore sentir aujourd'hui.

À cause de ses conséquences durables, il nous a semblé important de nous attacher ici à caractériser la période comprise entre 1660 et 1780 , en matière de traduction française de la tragédie grecque, et d'en préciser les enjeux. 


\section{Confusion entre traduction et adaptation au temps du classicisme}

On est frappé par le foisonnement des traductions qu'ont connu les débuts du classicisme en France. Les traductions du passé sont perçuess comme illisibles en un temps où l'exigence majeure est la suppression de la distance qui sépare le lecteur français du texte original, l'illusion d'un temps aboli, d'une culture universelle, mais qu'on veut en tous points semblable à celle qui se construit ici et maintenant.

La première conséquence de cet état d'esprit est le recul de la traduction des textes poétiques, trop intimement liés à la culture et au langage d'où ils procèdent. C'est pourquoí, consécutivement à l'abandon de la fidélité littérale, et à celui de la prétention poétique de la traduction, la traduction en prose gagne du terrain. Giry traduit l'Apologétique de Tertullien en 1636, Perrot d'Ablancourt traduit l'Octavius de Minicius Felix en 1637, Du Ryer, Cicéron, Les Philippiques en 1639, Hobier, Tacite, La vie d'Agricola, en 1639, etc. Les œuvres traduites sont surtout des œuvres d'éloquence ou des œuvres d'histoire (Zuber, 1995, p. 73-77).

Tous ces traducteurs semblent animés par la confiance d'une culture qui, sûre d'elle-même, n'hésite pas à prêter à d'autres ses moeurs parce qu'elles lui semblent plus élégantes, son esprit et sa manière de parler parce qu'elle les croit plus délicats. Le mépris que les traductions du XVI siècle des tragédies grecques inspirent aux critiques de l'époque classique se confond finalement avec le jugement que cette époque porte à l'égard de la culture étrangère elle-même, une culture qu'il faut surpasser, qu'il faut embellir.

Et si la tragédie ancienne n'est plus traduite pendant 120 ans - de 1572 (Baif, 1572), à 1692 (Dacier, 1692) - c'est, au fond, que le propos du traducteur français se confond avec celui de l'adaptateur : substituer les idées qui plaisent aujourd'hui à d'autres idées qui plaisaient au temps du poète. À quoi bon traduire Eschyle, Sophocle, ou Euripide, puisque les auteurs dramatiques rendent la vie aux mythes anciens, en les adaptant à la fois aux mœurs et aux formes dramatiques nouvelles? L'idee d'imitation regroupe en un même concept la traduction et l'adaptation. Devant l'Académie, en 1636, sous le règne de Louis XIII, Colleret fait l'éloge de l'imitation : " Que pour être éloquent, il faut imiter les Anciens, et qu'en les imitant, on peut les surpasser " (Colleret, 1636). À partir du moment où la 
tragédie a conquis en France son autonomie et son public, à l'âge baroque, la traduction de la tragédie grecque à fondement littéral et philologique se replie dans la langue latine, vecteur privilégié du savoir, comme en témoignent la réédition du dictionnaire grec-latin d'Henri Estienne (Estienne, 1611), et la trađuction latine complète du théâtre grec, due à Pierre de la Rovière (La Rovière, 1610).

\section{La traduction d'information générale à la fin du XVII' siècle}

Le véritable coup d'arrêt théorique à cette confusion des genres sera donné par Pierre Daniel Huet dans son traité en latin De optimo genere interpretandi (Huet, 1661). Huet rappelle le traducteur à ses devoirs de fidélité à la lettre de l'original :

[...] innatum unicuique sui ipsius amorem, bonarum rerum fere conjunctum, quum non auctorem modo auctorisque verbis subjectam sententiam respicit interpres, sed vel lectoris etiam servit voluptati aut studiis, vel suo ipsius genio indulget. [...] Dum enim de se bene existimat, et nimium sibi placet, judicis sibi facile arrogat partes, deque auctore ad quem convertendum accesserat, confidenter arbitrium facit. Quaecumque ergo sibi non sapiunt, respuit, proque iis sua supponit. Si quae vero etiam puncto ipsius comprobata sunt, eo tantum omine retinentur, si ita interpreti fuerit visum, qui mentis suae foetus in alienum nidum serpere et legitimos saepe exigere patitur. [...]

Meminisse debet interpres se determinatis circumclusum finibus, extra quos evagari non liceat : se alteri mancipatum, arbitrio suo morigerari non posse, sed totum ex alieno nutu pendere.[...] Auctoris formam ita induat ut totus ipse sub aliena specie delitescat, et quotiescunque oculos lector vel perspicacissimus intenderit, auctorem cernat, ipse interpres nusquam appareat. [...] Sic enim existimo, quicunque interpretis suscipit partes, in eo praecipue ipsius eniti dbere industriam, non ut facultatem dicendi, si qua forte praeditus est, exerceat, et orationis suavitate auribus fucum faciat : sed ut anctorem, tanquam in speculo et imagine, sic in verbis suis contuendum exhibeat, adscitumque omnem ornatum detrahat.

Quum enim nihil aliud esse videatur interpretatio, quam expressa auctoris imago et effigies, id perfecto efficitur, eam demum praestabiliorem esse interpretationem, non quae auctoris vel luxuriem depascat, vel jejunitatem expleat, vel obscuritatem illustret, vel menda corrigat, vel perversum ordinem digerat; sed puate totum auctorem ob oculos sistat nativis adumbratum coloribus, et vel suis virutibus laudandum, vel, si ita meritus est, propriis deridendum vitiis propinet. 
[...] l'amour inné de chacun pour soi-même, auquel se joint une absence quasi totale de bon sens, quand non seulement le traducteur ne tient compte ni de l'auteur ni de la phrase déterminée par les mots de l'auteur, mais au contraire tantôt se fait l'esclave du contentement et des goûts du lecteur, tantôt s'abandonne à sa propre inspiration.[...] Tout en cultivant de lui-même une haute opinion et une complaisance immodérée, il s'arroge aisément le rôle de juge et se fait hardiment l'arbitre d'un auteur qu'il s'était proposé de traduire. C'est ainsi qu'il rejette tout ce qui manque de saveur selon lui, pour y substituer de son propre cru. Mais si quelque chose a mérité son approbation, il l'accepte pour ce seul augure, traducteur ainsi fait que s'il a matière à satisfaction, c'est qu'il admet que les productions de son esprit se glissent dans le sein d'un autre, et qu'il les exige souvent conformes à ses lois. [...]

Le traducteur doit se rappeler qu'il est enfermé dans les limites déterminées en dehors desquelles il ne lui est pas permis de s'échapper; qu'il est tout aux mains d'un autre, qu'il ne peut pas etre complaisant à son propre arbitre, mais qu'il dépend entièrement de la volonté d'un autre. [...] Qu'il revête la forme de l'auteur de telle façon que lui-même tout entier se cache dans l'aspect d'un autre, et qu'à chaque fois que le lecteur même le plus perspicace a concentré son attention, qu'il disceme l'auteur, le traducteur, lui, n'apparaisse jamais. [...] Ainsi je pense en effet que l'art de celuj-là même qui, quel qu'il soit, assume la fonction de traducteur, doit tendre en premier lieu non pas à exercer le pouvoir de la parole, si le hasard l'en a pourvu, ni à flatter les oreilles par la douceur du discours, mais à montrer qu'il faut s'attacher à l'auteur, à travers les mots qui sont les siens, comme dans un miroir ou sur un portrait, et à proscrire tout omement rapporte. [...]

Car si la traduction ne semble être rien d'autre que l'image et l'effigie exprimées de l'auteur, il s'ensuit assurément que la seule traduction qui soit de plus grande valeur est celle qui, loin d'entamer la profusion de l'auteur, ou de suppléer à sa maigreur, loin d'éclairer l'obscurité, de corriger les defauts ou de rectifier un ordre maladroit, au contraire fait paraitre à la vue l'auteur tout entier, le reproduit en ses couleurs originelles et destiné, soit à être loué pour ses vertus, soit, s'il l'a mérité, à êtré raillé pour ses défauts mềmes.

${ }^{1}$ Cette traduction littérale, comme les suivantes, sauf indication contraire, est de l'auteur de l'article. 
Huet, pour rendre compte du rôle du traducteur, adopte l'image du portrait fidèle ( $a$ auctoris imago et effigies $»)$. Cette image restreint considérablement le rôle du traducteur, qui doit s'effacer derrière l'auteur, (" sub aliena specie delitescat *), ne jamais apparaître (" nusquam appareat "), et se soumettre entièrement à la volonté de l'auteur (* ex alieno nutu pendere $n$ ). Cependant, l'heure n'est pas, en France, au temps du classicisme, à la fidélité littérale, ni à la restitution des figures poétiques des textes sources. Le traducteur, soumis à la volonté de l'auteur qu'il traduit, conformément au précepte de Huet ( Ex alieno nutu pendere ), ne se sent pas pour autant assujetti à l'articulation poétique et littérale du texte à traduire.

André Dacier, dans la prêface de la traduction de l'Électre de Sophocle, parue à la fin du XVII' siècle, affirme en ce sens qu'il lui faut renoncer à la restitution de a la diction *. Tout en admettant l'importance de la forme poétique, il préfere renoncer à la rendre. * La tragédie, écrit-il, faisant son imitation par le discours, le nombre et l'harmonie, [...] ne doit être qu'en vers ", mais le traducteur préfere rendre seulement * ce qui plaît à l'esprit „, et compare le résultat obtenu à a l'ébauche d'un tableau dont tous les contours sont bien arrêtés, toutes les parties bien dessinées, où il ne manque que l'éclat des couleurs " (Dacier, 1692, "préface "). Ce renoncement du traducteur à rendre la globalité des effets poétiques du texte qu'il traduit doit être compris comme la limitation de son travail à la restitution de l'idée, (* ce qui plaît à l'esprit ", dit Dacier), seul élément contenant une part d'universalité, parce que relevant de la raison humaine, et non de la langue et de l'époque du traducteur.

Le développement des notes explicatives, dans les traductions de l'époque du classicisme, permet au traducteur de rendre compte de ce qu'il considère ne pas devoir ou ne pas pouvoir traduire : 216 remarques scène par scène suivent le texte de la traduction de l'CEdipe Roi de Sophocle par Dacier. L'exemple de sa traduction des vers $1227-1231$ nous fournira une illustration de cette manière de traduire. Le messager, avant d'annoncer la mort de Jocaste, se livre à une réflexion générale sur l'ampleur des maux qu'on s'est attirés volontairement :

\section{EE.ATTE $\Lambda O \Sigma$}

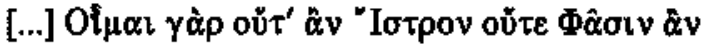

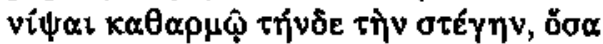




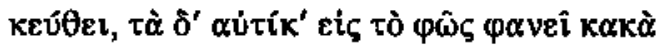

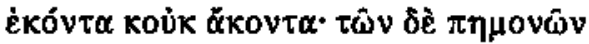

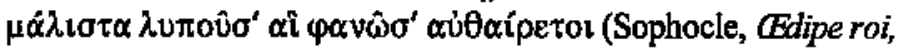
1985 , v. 1227-1231).

\section{Traduction française :}

\section{Le Messager}

Je pense en effet que ni l'Ister ni le Phase ne laveraient par purification cette maison-ci, quant à tout ce qu'elle cache, mais elle va montrer à la lumière les maux voulus, et qui ne sont pas non voulus; or entre les peines, celles qui affligent le plus sont celles qui de toute évidence ont été choisies.

Lisons la traduction de ce passage et la note complémentaire de Dacier :

\section{L'Ecuyer :}

Je ne crois pas que les eaux du Danube, ni celles du Phase puissent jamais laver les taches de cette maison; vous allez voir de vos propres yeux les maux qu'on vient d'y commettre de propos délibéré; les plus sensibles de tous les malheurs sont ceux que l'on paroit s'attirer volontairement soi-mème.

\section{Note :}

II y a des malheurs qui nous arrêtent sans que nous les attirions, et ce sont les malheurs qu'on appelle $"$ involontaires *. Nous pouvons trouver en nous assez de force pour les supporter; tels sont les premiers malheurs d'CEdipe. Il y en a d'autres qu'on appelle a volontaires", et que nous attirons comme à dessein, tels sont les demiers malheurs de ce prince; il s'opiniâtre à se chercher lui-même, malgré les efforts de ceux qui lui donnent un meilleur conseil : il s'arrache les yeux et se rend l'homme du monde le plus misérable. Sophocle dit que ces derniers malheurs sont plus insupportables que les premiers et il a raison. II n'y a rien de si sensible que le mal que l'on s'attire par sa faute; car à la douleur se joint le reproche et le repentir, toujours plus insupportables que la douleur même. Voilà en quoi Sophocle suit l'opinion commune; mais comme en remontant plus haut, on trouve que tout est la suite et l'effet d'une providence réglée qui conduit le monde, et qui ne laisse rien arriver au hasard, ce Poète pour se conformer à cette vérité, sans blesser l'opinion du peuple, dit " que l'on paroit s'attirer volontairement soi-même ". Cela mérite d'être remarqué. (Dacier, 1692, p. 123-124 et 228) 
Pour un lecteur moderne, le passage offre une indéniable résistance à la compréhension. Le messager désigne le palais d'EEdipe comme étant souillé de maux, et on ne peut songer qu'à la malédiction divine qui frappe les Labdacides, et dont un oracle, dans la pièce de Sophocle, a rappelé qu'il poursuivait en CEdipe, dès sa naissance, celui qui allait tuer son père. Mais aussitôt le messager affirme que ces malheurs sont volontaires, et qu'on ne souffre jamais tant que des maux choisis volontairement. Quelle est donc la responsabilité d'Edipe dans le malheur qui le frappe? Voilà bien ce que le traducteur voudrait savoir, mais précisément c'est une question que Sophocle ne pose jamais en ces termes. Comme l'a noté Jacqueline de Romilly, " on peut remarquer que, dans Edipe roi, on ne s'interroge jamais sur la raison de ce qui arrive à Edipe et devait lui arriver. Sophocle ne cherche nullement à expliquer la sévérité du sort fait à CEdipe [...]. Il n'y a rien à expliquer, aux yeux de Sophocle : il n'y a pas d'explication, mais pas non plus de question. Les choses sont ainsi, tout simplement n (Romilly, 1992, p 110). Un passage tel que celui-ci présente un véritable défi à l'homme cartésien. Pourtant dans le théâtre grec antique, même lorsque les événements sont présentés comme issus d'une décision divine, la responsabilité de l'homme n'est pas niée (Romilly, 1992, p. 171-172). Dans (Edipe roi en particulier, les deux niveaux coexistent : le destin d'origine divine ( $\delta \alpha \imath^{\prime} \mathrm{E} \mu \omega v$, Sophocle, 1985, v. 1311), qui poursuit la race des Labdacides, et que matérialise la maison

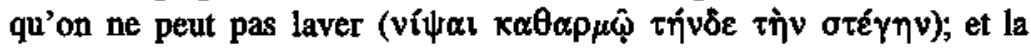
responsabilité d'Edipe qui méprise les oracles et outrage les dieux. L'hamartia, qui désigne cette faute en grec (voir Saïd, 1978), n'implique sans doute ni vice ni perversion morale, mais plutôt une transgression de la norme, un dépassement de la mesure, que les Grecs nommaient l'hybris (voir Edipe roi, Sophocle, 1985, v. 873-877). C'est pourquoi, dans le passage qui nous occupe, les malheurs d'ECdipe peuvent être présentés comme la conséquence de son erreur, le résultat manifeste de sa volonté, ainsi que l'indique le médio-passif $\varphi \alpha v \omega \hat{\omega} \sigma^{\prime}$, " se montrer manifestement ": Edipe aurait pu agir autrement, et respecter pieusement l'oracle divin. Mais en dépassant la mesure, il s'est attiré les maux les plus attristants qui soient, ceux qui sont, de toute évidence, le résultat d'un libre choix.

André Dacier traducteur de Sophocle ne peut pas se ranger à cette vision antique de la faute tragique. Dans le détail, sa traduction comporte trois inflexions par rapport au texte de Sophocle (voir Biet, 1982, p. 133135). Les deux premières relèvent de la correction stylistique, qui doit 
respecter la norme de la prose d'art française : ce n'est plus la maison qu'il faudrait laver de toute souillure, mais les maux eux-mêmes (comparer

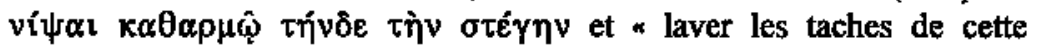

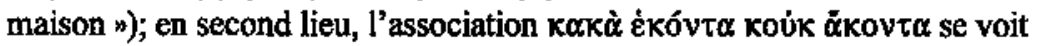
privée de son second terme considéré comme redondant, puisqu'il s'agit d'une double négation (" les maux voulus et non involontaires »). La troisième inflexion a une toute autre motivation, elle change le sens du texte et fait l'objet de l'explication en note : le traducteur, en choisissant, pour rendre le grec $\varphi \alpha v \hat{\omega} \sigma^{\prime}$, le verbe * paraître » au sens de "avoir l'air de ", réduit la participation de la volonté de l'homme dans son malheur à une vue de l'esprit, à une appréciation subjective.

Pour Dacier, il faut résoudre la contradiction qu'il croit trouver chez Sophocle : comment Sophocle pourrait-il parler d'un mal qu'on s'est attiré volontairement, si un implacable destin détermine le malheur des hommes (* une providence réglée qui conduit le monde, et qui ne laisse rien arriver au hasard n)? Puisque la fatalité est toute-puissante, l'homme se trompe se croyant la cause de ses malheurs. Il croit ainsi qu'il s'attire volontairement le malheur qu'il paraît s'être attiré, mais, en réalité, c'est la fatalité d'origine divine qui en est la cause.

Dacier donne donc une valeur psychologique à l'idée : il met Sophocle du côté de la pensée du temps du classicisme français, croyant trouver en lui une vérité générale, explicitée en note, dans laquelle il se reconnaît : « il n'y a rien de si sensible que le mal que l'on s'attire par sa faute; car a la douleur se joint le reproche et le repentir, toujours plus insupportables que la douleur même *. Cette vérité générale est un thème présent chez les moralistes du XVII ${ }^{e}$ siècle. Deux maximes de La Rochefoucauld en font foi :

Il faut demeurer d'accord, à l'honneur de la vertu, que les plus grands malheurs des hommes sont ceux où ils tombent par leurs crimes.

Les violences qu'on nous fait nous font souvent moins de peine que celles que nous nous faisons à nous-mêmes (La Rochefoucauld, $1665, \mathrm{n}^{\circ} 187 \mathrm{et}$ 385).

André Dacier préfêre mettre à jour une vérité générale que ses lecteurs vont comprendre, plutôt que s'en tenir aux mots qu'il trouve dans le texte source. Témoignent de cette démarche l'usage de la première personne du pluriel dans sa note ( Nous pouvons trouver en nous assez de force pour les 
supporter... n), qui abolit la distance entre le temps du texte source et le temps du traducteur, et l'expression par laquelle Dacier approuve Sophocle, ("Sophocle dit que [...] et il a raison "), qui met le traducteur et l'auteur sur le même plan, tous deux à la recherche d'une vérité générale. C'est avec les idées morales du temps du classicisme que Dacier tente de rendre compatible le texte qu'il traduit. Le texte est tiré vers les caractères * universels " dont les moralistes du XVI" siècle se sont faits les théoriciens. Cependant, le traducteur peut se déclarer soumis à la volonté de l'auteur et de fait il souhaite la faire comprendre à son lecteur. Mais faire comprendre signifie, pour lui, rendre vie aux idées universelles de l'esprit humain qu'il croit y découvrir, et non partir à la recherche d'une différence, restaurer une distance, laisser paraître l'historicité du texte.

En donnant à la traduction une méthode rationnelle et des ambitions limitées, qui la distinguent de l'imitation, le dernier quart du siècle du classicisme, passant de la querelle des anciens et des modernes a l'analyse des œuvres (Fontenelle, 1688), réclame des traductions françaises fidèles tout au moins au sens, et susceptibles de donner au lecteur une information intelligible et clairement exprimée. C'est pourquoi nous nommerons cette famille de traduction la Traduction d'information gênérale.

Cette forme de traduction convient alors aux attentes des lecteurs. La prose d'art, respectueuse des normes stylistiques françaises, peut estomper les métaphores de l'original et ignorer ses formes poétiques. L'interprétation, l'élimination des redondances ou des contradictions, sont bienvenues. On ne lui interdit qu'une chose : l'invention. Racine, se faisant traducteur d'Euripide pour l'information du lecteur de son Iphigénie, le prévient en des termes proches de ceux d'un Dacier, de son inaptitude à rendre la beauté de l'original : « J'aurais souhaité de pouvoir exprimer dans ces vers les grâces qu'ils ont dans l'original. Mais au moins en voici le sens * (Racine, 1674, p. 15). Les grâces de l'original ne sont pas niées, car l'érudition ne s'est pas éteinte, mais c'est une autre dramaturgie et une autre esthétique qu'il faut servir, dont l'Art poétique de Boileau s'emploie a faire l'aboutissement glorieux de l'histoire universelle du thêatre. Entre une adaptation qui plie les mythes de l'Antiquité aux structures de la dramaturgie nationale et une traduction soucieuse de donner aux lecteurs une information générale sur les idées déposées dans les æuvres des 
anciens, quelle place pourrait-il rester à une traduction poétique des tragédies grecques?

\section{Vers l'impossibilité théorique de la traduction poétique}

Le XVIII' siêcle a d'abord été le siècle de la réflexion critique sur la traduction, liée au développement de la philosophie du langage. Or cette réflexion, jusqu'aux années 1780 , aboutit le plus souvent au constat de l'impossibilité de la traduction fidèle des poètes.

Reprenant une fois encore la métaphore de la représentation picturale, l'abbé Jean-Baptiste Dubos, en 1719, réduit la part du traduisible non plus à l'idée de l'auteur, mais à l'organisation générale de l'œuvre : " Une traduction est une estampe où rien ne demeure du tableau original que l'ordonnance et l'attitude des figures ». L'argument de Dubos s'appuie sur une définition perceptive de l'œuvre littéraire : sa valeur n'est plus liée, comme c'était le cas dans la préface de l'Électre traduite par Dacier, aux idées universelles qu'il faut identifier et faire comprendre, mais aux émotions qu'elle suscite en nous. Le poème, écrit-il, nous touche « par la voie du sentiment *. Et c'est au sentiment " qu'il appartient de connaître si l'objet qu'on nous présente est un objet touchant et capable de nous attacher, comme il appartient à l'oreille de juger si les sons plaisent, et au palais si la saveur est agréable * (Dubos, 1719, p. 477-498).

Il n'est plus question de faire comprendre les seules idées présentes dans l'esprit de l'auteur, avant leur mise en mots, mais de créer les mêmes effets sur les sens du lecteur que ceux que pouvait susciter l'original. Pour y parvenir, il faut que le traducteur * se donne la liberté de changer les figures et d'en substituer d'autres qui sont en usage dans sa langue $\times$ (Dubos, 1719 , p. 477-498). On songe ici à Diderot : « Il n'y a $q u^{\prime} u n$ moyen de rendre fidèlement un auteur $d$ 'une langue étrangère dans la nôtre : c'est d'avoir l'âme pénétrée des impressions qu'on a reçues et de n'être satisfait de sa traduction que quand elle éveillera les mêmes impressions dans l'âme du lecteur. Alors l'effet de l'original et celui de la copie sont les mêmes. Mais cela se peut-il? " (Diderot, 1762, tome V, p. 236). Par anticipation, Jean-Baptiste Dubos répondait négativement à cette question de Diderot : $\alpha$ [...] j'accuse les critiques de n'avoir point assez de connaissance des mours et des usages des différents peuples pour juger quelles figures ils autorisent ou n'autorisent pas * (Dubos, 1719, p. 477- 
498). Diderot lui-même, dans un autre texte, ne se montre pas plus optimiste que Dubos:

Je croyais, avec tout le monde, qu'un poète pouvait etre traduit par un autre : c'est une erreur et me voilà désabusé. On rendra la pensée; on aura peut-être le bonheur de trouver l'equivalent d'une expression [...], c'est quelque chose, mais ce n'est pas tout. L'emblème délié, l'hiéroglyphe subtil qui règne dans une description entière, et qui dépend de la distribution des longues et des brèves [...] dans les langues à quantités marquées, et de la distribution des voyelles et des consonnes dans les mots de toute langue, tout cela disparait nécessairement dans la meilleure traduction [...]. Sur cette analyse, j'ai cru pouvoir assurer qu'il était impossible de rendre un poète dans une autre langue; et qu'il était plus commun de bien entendre un géomètre qu'un poète (Diderot, 1751, vol. I, p. 376).

Si l'on ne peut pas trouver d'images équivalentes à celles de l'original, ne pourrait-on pas, en s'en tenant au mot à mot, traduire fidèlement toutes les figures qui s'y trouvent? C'est, pour Dubos, un échec * plus grave encore * que la recherche d'images de substitution dans sa langue. Car l'impression que produit la figure, dans l'original, ne peut plus être suscitée par une traduction littérale dans une autre langue : * " manger son pain à l'ombre d'un figuier " faisait impression sur un Syrien persécuté par un soleil ardent " (Dubos, 1719, p. 477-498), mais il n'en va plus de meme sur le lecteur français d'aujourd'hui.

Dubos aboutit donc à la conclusion de l'impossibilité de la traduction des poètes. Il n'accorde finalement à la traduction d'autre intérêt que l'instruction de l'historien. Les beautés de l'original lui sont interdites. Le travail de restitution de la " forme " (toujours séparée de l'esprit) est voué à l'échec.

Pourtant, la question de la possibilité de la traduction des poètes, si elle aboutit provisoirement à la même conclusion pessimiste que celle qu'on trouve sous la plume de Dacier, a le mérite d'avoir été posée en des termes nouveaux, qui laissent entrevoir d'autres possibilités de réponse, d'autres manières de traduire que l'explicitation d'un * sens * compris avec les structures rationnelles du temps du traducteur. Les idées de Dubos ne sont d'ailleurs pas passées inaperçues. L'Abbé Desfontaines le pille abondamment, sans nommer son devancier, dans un ouvrage de critique 
littéraire du milieu du $\mathrm{XVIII}^{\circ}$ siècle (Desfontaines, 1757), qu'on citera encore au siècle suivant (Vaultier, 1812).

À partir du dernier quart du XVIII ${ }^{\mathrm{e}}$ siècle, la condamnation de la traduction des poètes est controversée, sur des bases pourtant inchangées depuis Dubos. Mais on semble vouloir passer du débat théorique à l'expérimentation. Les deux modes de traduction présentés en théorie par l'abbé Dubos sont tour à tour expérimentés en fin de siècle : la reconstitution des effets de l'original par ume liberté d'invention, (que nous nommerons ici Traduction-imitation), d'une part, et d'autre part la fidélité rigoureuse aux termes de l'original, en prose (Traduction analytique en prose).

\section{Le développement de la Traduction analytique en prose}

La Traduction analytique en prose naît, à la fin du XVIII siècle, de la critique que suscitent les Traductions d'information générale du début du siècle. On reproche au Père Brumoy, l'auteur de la première anthologie française de traductions des trois poètes tragiques grecs de l'Antiquité (Brumoy, 1730), les inflexions qu'il fait subir au texte des anciens. Belin de Ballu, traducteur d'Hécube à la fin du XVIII' siècle, reproche au Père Brumoy d'adapter le texte d'Euripide dans un sens chrétien, en particulier quand il atténue les marques d'athéisme du dramaturge grec. A propos de la traduction des vers 488-491, il note : « Le père Brumoy a défiguré ce passage tant qu'il a pu. L'idée d'athéisme qu'il présente l'a sans doute effrayé. Mais un traducteur n'est pas responsable des opinions de son auteur " (Euripide, 1783, note au vers 491). Un traducteur anonyme de la même époque fait la même remarque au même endroit : * Le Père Brumoy effrayé de l'athésisme que présente cette pensée l'a entièrement déguisée * (Euripide, s.d., note au vers 491).

Trois traducteurs de cette époque, qui se rattachent à la catégorie des Traductions analytiques en prose, à savoir Belin de Ballu, Prévost, et le traducteur anonyme de la fin du XVIII ${ }^{\mathrm{e}}$ siècle, se rejoignent en maints autres endroits de l'Hécube d'Euripide pour adresser les mêmes reproches à la version de Brumoy.

Par exemple, Brumoy avait fait disparaître de la traduction du début de la déclaration de Polyxène à Ulysse, (v. 342-378), le geste rituel 
des suppliants, touchant le menton du supplié. Il s'agit pourtant seulement d'un geste, qui, bien qu'un peu étrange, ne présente pas de difficulté de traduction. Mais ce même texte d'Euripide comporte une expression autrement plus difficile à traduire : Polyxène dit à Ulysse qu'il a échappé à "[s]on Zeus suppliant ", ce qui n'est pas seulement étrange, mais incompréhensible en français. On mesurera, à la manière dont nos traducteurs ont contourné ces deux embaches, les limites des droits et des devoirs que chacun d'eux accorde au traducteur :

\section{IIOAYEENH}

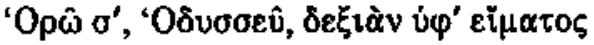

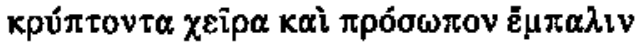

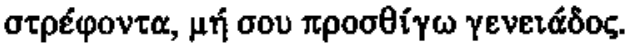

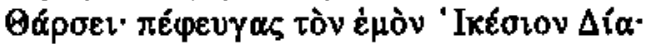

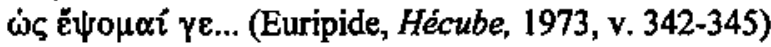

\section{Traduction française :}

\section{Polyxène}

Je te vois, Ulysse, cacher la main droite sous le manteau, et détourner en arrière le visage, de peur que je ne touche ton menton. Rassure-toi : tu as échappé à mon Zeus suppliant; de sorte que je te suivrai assurément.

Brumoy, 1730 :

Je le vois, Ulysse, vous cachez votre main, vous détoumez le visage. (1) Vous redoutez mes prières; ne craignez rien : vous n'entendrez de moi ni voux ni soupirs. Je vous suis.

Note du traducteur :

(1) C'était pour empêcher qu'on ne lui touchât la main ou le menton, coutume des suppliants.

Belin de Ballu, 1783 :

Ulysse, je vous vois cacher votre main sous votre vêtement; vous détournez le visage, de peur que je ne vous touche au menton. Rassurez-vous. Vous ne me verrez point descendre au ton de suppliante. Je suis prête à vous suivre.

Note du traducteur :

Le grec dit " vous avez evitez mon Jupiter Suppliant ". C'est une formule de langage qu'il fallait changer; mais elle est piquante, et le lecteur ne 
doit pas la perdre; d'ailleurs elle apprend que Jupiter pressidait aux supplications.

Prévost, 1785 :

Je vous vois, Ulysse, cacher votre main droite sous vos vêtements, et détourner votre visage de peur que ma main ne l'atteigne. Rassurez-vous; vous n'avez point à craindre le Dieu vengeur de mes supplications : me voici prête à vous suivre.

Note du traducteur :

Littéralement $\alpha$ vous avez échappé à mon Jupiter suppliant . On se rappelle ici la coutume antique des suppliants, de porter la main au menton ou à la barbe.

Brumoy ne traduit aucune des deux difficultés repérées : ni le geste rituel,

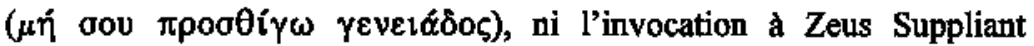

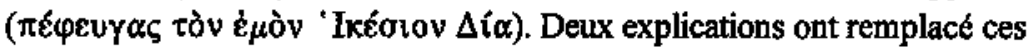
détails, pour l'information du lecteur : * vous redoutez mes prières ", qui restitue l'idée générale que le traducteur extrait du geste rituel, et * vous n'entendrez de moi ni vœux ni soupirs *, pour représenter l'idée d'une supplication. Le geste du rituel fait l'objet d'une mention en note.

Les deux traducteurs de la fin du siècle marquent un progrès de précision : tous deux traduisent le geste du rituel : * de peur que je ne vous touche au menton ", dit Ballu, et $\alpha$ de peur que ma main ne l'atteigne [votre visage] ", chez Prévost, qui complète en note la partie du visage touchée (" On se rappelle ici la coutume antique des suppliants, de porter la main au menton ou à la barbe ). En revanche, la traduction de la supplication de Zeus les embarrasse toujours autant que Brumoy. La difficulté, on l'a vu, est d'un autre ordre : " mon Zeus suppliant " n'a aucun sens en français. Le possessif « mon » et le qualificatif « suppliant * ne s'appliquent pas au nom du Père de l'Olympe sans créer une obscurité en français. Ballu traduit l'idée, et réserve le détail pour la note, tandis que, cette fois, Prévost est plus précis. Il a l'habileté de restituer l'invocation divine, le possessif, et l'intention suppliante, dans une périphrase : * vous n'avez point à craindre le Dieu vengeur de mes supplications". Cependant, la note restitue la version littérale. Voilà le lecteur parfaitement au fait de l'idée et de sa formulation. Mais en définitive, lui a-t-on véritablement proposé une traduction? 
La méthode de traduction qui naît au cours du XVIII' siècle, la Traduction analytique en prose, décompose le texte en unités de signification, et les répartit, suivant leur degré de compatibilité avec les usages littéraires français, soit dans le corps de la traduction, soit dans les notes. Les notes jouent le rôle d'un débarras commode pour tout ce qui paraît trop éloigné de la culture du lecteur. Un glissement semble s'établir, entre la traduction de Brumoy et celles de ses successeurs en fin de siècle, du non traduit vers les notes et des notes vers le corps de la traduction.

Les traductions analytiques ont une solide assise philologique, et leurs auteurs n'hésitent pas à comparer les leçons des scholiastes pour établir le texte. Certaines notes en témoignent. Par exemple, à propos du vers 53 de l'Hécube d'Euripide, notre traducteur anonyme suit la proposition du scholiaste qui s'était étonné qu'Hécube sortît de la tente

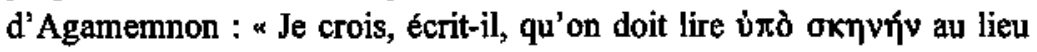

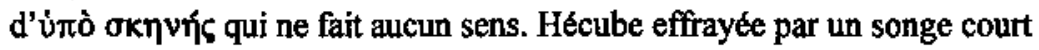
à la tente d'Agamemnon pour y consulter sa fille Cassandre captive de ce prince et qui avait reçu d'Apollon le don de divination. "(Euripide, s.d., note du vers 53). Le souci de précision des traducteurs analytiques doit donc être rapproché du développement progressif de l'érudition. Les débuts de la paléographie ne remontent en effet qu'à la fin du XVII ${ }^{e}$ siècle. Auparavant, si la critique textuelle avait fait des progrès, on ne s'intéressait guère à la date et à l'origine des manuscrits utilisés pour les éditions des textes classiques. Dom Bernard de Montfaucon (1655-1741), publia en 1708 la Paleagraphia graeca, créant le néologisme qui désigne depuis lors cette discipline nouvelle. Dans le même temps, on découvrit que des textes classiques pouvaient être encore dissimulés dans l'écriture inférieure des palimpsestes. L'intérêt nouveau pour l'origine du texte grec et son établissement rejaillit sur la recherche de précision de sa traduction française.

Plus généralement, il y a derrière ces choix communs de traduction en fin de siècle la prise de conscience d'une loi de traduction, une sorte de déontologie du métier de traducteur. Ballu, Prévost, et le traducteur de l'Hécube resté anonyme ont intériorisé la relativité des cultures, le respect de l'altérité, et l'honnêteté intellectuelle qui consiste à tout transmettre au lecteur. Les détails concrets, qu'ils n'osent pas tous traduire, peuvent intéresser le lecteur, car ils témoignent d'une culture et d'un temps différents des nôtres. Il y a une curiosité pour les faits de civilisation et pour 
la comparaison des cultures dans ces ouvrages. La réédition des Antiquitates graecae et romanae de Montfaucon en 1757 témoigne, parmi d'autres ouvrages de cette époque, de cet intérêt pour les faits de civilisation.

Les attentes du public ont changé. Parlant de l'auteur d'Hécube, Ballu résume en ces termes cet intérêt nouveau : * Nous ne nous sommes permis d'altérer aucune de ses pensées, sous le spécieux prétexte qu'elles répugnaient à nos mœurs et à nos usages. [...] Ce sont ces traits réunis et bien exprimés qui forment le costume, le caractère original qui distingue un Écrivain étranger et lui donnent cette physionomie particulière dont la nouveauté attache et pique un Lecteur amoureux de l'Antiquité " (Belin de Ballu, 1783, " Avertissement "). L'cuvre traduite n'est pas rapportée à l'expression d'une poésie singulière, mais au témoignage d'une époque et d'une culture lointaines. « Peut-être, écrit encore en ce sens Prévost en 1785, au moment où écrivait ce judicieux appréciateur des beautés antiques, [le Père Brumoy], le public ne pouvait-il en supporter l'éclat : trop accoutumé à juger les ouvrages dramatiques sur des modèles plus récents, ce public poussait à l'excès la crainte de voir violer les convenances qu'il y remarquait. [...] La lecture plus répandue des ouvrages écrits dans les langues étrangères, a rendu peut-être le goût moins exclusif, les lumières de la philosophie ont donné aux esprits plus d'étendue et de hardiesse, enfin le temps et la méditation, ces deux arbitres de l'opinion, ont travaillé pendant un demi-siècle à ramener insensiblement à cette heureuse simplicité, dont les plus grands maîtres de la scène française avaient été contraints de s'écarter pour ne point blesser leurs contemporains " (Prévost, 1785, vol. 4, * Avertissement ").

Le témoignage de Prévost présente le mérite de situer la traduction par rapport à son public, et d'établir sans ambigulté la dépendance du traducteur à l'égard de son lectorat. La traduction jésuite du Père Brumoy avait conrespondu à une forte demande, et y avait répondu au-delà de toute espérance : aucun autre traducteur français n'avait pu détrôner cette bible française du theâtre grec pendant plus d'un demi-siècle. Trente ans après la première édition, un autre Père Jésuite, J. Fleuriau, la réédite sans changer une virgule, mais en y ajoutant des notes infra-paginales. L'autorité de cet ouvrage, inscrit dans une perspective pédagogique, en fait à la fois un manuel de littérature ancienne à l'usage des collèges jésuites français - où le latin, signe des temps, est peu à peu supplanté par le français comme 
langue véhiculaire de l'Antiquité -, mais aussi un moyen d'accès rapide au thêâtre des Grecs pour un public plus large, désireux de savoir ce qu'était ce theatre, et ne se contentant plus des adaptations dramatiques modernes, trop modernes, sans doute. Le goût pour les anciens ne se réduit plus à la transposition theatrale ou romanesque des mythes de l'Antiquité, il demande une connaissance des œuvres de l'Antiquité.

Ce mouvement s'est suffisamment amplifié pour qu'en fin de siècle le travail de polissage des aspérités grecques, opéré avec tant de patience par le Père Brumoy dans sa Traduction d'information générale, ne convienne plus à un public qui sait à présent reconnaître dans cette traduction les formes et les idées du théâtre et de la litterature de son temps, et qui s'en plaint. C'est pour répondre à cette nouvelle demande de précision, de fidélité littérale, qu'est réalisée cette entreprise de grande envergure qu'est la réddition du Théâtre des Grecs du R. P. Brumoy, et qui ne peut se justifier que par un mouvement très prononcé en faveur du thêâtre antique. Qu'on ne s'y méprenne pas : il ne s'agit pas d'une simple réédition, mais d'une refonte complète, menée par une équipe de traducteurs, une édition de vulgarisation, sans doute, mais à destination d'un public exigeant. Charles Brottier s'est chargé du thêâtre d'Aristophane, La Porte du Theil, d'Eschyle, Rochefort, de Sophocle, et Prévost, d'Euripide.

Cette édition monumentale ne détruit pas les strates des éditions antérieures du Théâtre des Grecs du Père Brumoy : elle en ajoute une autre, qui se présente comme une critique, qui, à travers les notes et les préfaces des unes et des autres, se compose d'interpellations, de jugements, de contradictions. Comme l'annonce le traducteur d'Euripide dans son avertissement, " L'obligation qu'on s'est imposée de réimprimer sans le moindre changement le texte du Père Brumoy, impose en même temps celle de le réfuter quelquefois " (Prévost, 1785, vol. 4, " Avertissement n). Aux notes de l'édition de 1730 , qui se repèrent à l'aide de signes diacritiques (l'astérisque par exemple), s'ajoutent celles de la réédition de 1763, reconnaissables à l'inscription * $\mathrm{AE}$ » (Ancien Éditeur), enfin complétées par les notes propres à l'édition de 1785 . Les notes dues au nouveau traducteur d'Hécube ne se limitent d'ailleurs pas à commenter Brumoy : elles citent et critiquent également les autres traductions contemporaines, celle de Belin de Ballu, et celle, partielle, de La Harpe. 
Synthèse de la traduction des tragiques grecs, l'édition du Théatre des Grecs de 1785 entend mettre un certain ordre dans l'édition de 1730. Soucieuse de vérité historique, elle replace les pièces dans l'ordre chronologique de leur composition. Mais surtout, elle assoit d'une façon durable la Traduction analytique en prose comme le meilleur mode de transmission du texte ancien pour qui veut comprendre l'Antiquité. Le succès de cette édition de 1785 est prouvé notamment par cette édition bilingue d'Hécube de 1829, qui comporte le texte grec d'Hécube et deux traductions françaises, l'une littérale et interlinéaire, * avec la construction du grec dans l'ordre naturel des idées ", et une autre, en français rédigé (Euripide, 1829). Cette dernière version n'est que la réédition de la traduction de Prévost de 1785 rarement modifiée par Ph. Lebas, qui note dans son introduction : " On s'est servi dans cette édition de la traduction de Prévost qui a été revue avec soin \%. Plus tard encore, V. H. Chappuyzi, en 1836 (Euripide, 1836), et C. Leprévost, dans la version juxtalinéaire de 1901 (Euripide, 1901) publiée dans la collection des Auteurs Grecs de la librairie Hachette, utilisent encore largement, sans la citer, la traduction de Prévost.

Qu'on en juge par la comparaison de ces versions du début du premier stasimon d'Hécube (v. 444-450) distantes de plus d'un siècle :

Prévost, 1785 :

Vents qui soufflez sur l'océan, et qui portez les vaisseaux rapides sur les ondes enflées, où conduirez-vous mon infortune? Sous quel maître et en quejs lieux irai-je servir?...

Leprévost, 1901 :

Vents qui soufflez sur les mers, vents qui portez les rapides vaisseaux sur le dos gonflé de la plaine liquide, où conduirez-vous mon infortune? Sous quel maître, en quelle demeure irai-je servir?...

Les premières Traductions analytiques en prose du XVII' siècle préfigurent les traductions en prose que produiront le $\mathrm{XXX}^{\circ}$ siècle et les traductions scolaires et universitaires sur lesquelles nous travaillons encore aujourd'hui. Il est donc de la plus grande importance de repérer ces traductions dès leur origine, dans le dernier quart du XVIII ${ }^{\circ}$ siècle, et de ne pas les confondre avec les Traductions d'information générale, comme on le voit faire 
quelquefois sous la plume des auteurs qui se sont intéressés à l'histoire de la traduction à cette époque. Sous une appellation générique, on a dit des " successeurs de Brumoy " que dans leurs traductions, $~$ l'exactitude n'est pas de mise ", (Romilly, 1979, p. 79-95), en s'appuyant au besoin sur des exemples qui prouvent à l'évidence que les traductions citées n'appartiennent pas au genre des Traductions analytiques en prose. C'est ainsi que dans la traduction de Prométhée par Le Franc de Pompignan, (Eschyle, 1770), le taon qui poursuit Io (v. 566) devient " un nouvel accès

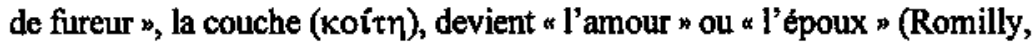
1979 , p. 79-95). On ne peut pas ainsi confondre toutes les traductions de tragédies grecques du XVIII' siècle dans le même moule de l'infidélité littérale. La situation de la traduction de la tragédie grecque nous semble plus complexe et moins uniforme. Trois catégories de traductions existent à cette époque.

Premièrement la Traduction d'information générale, qui est la plus ancienne, puisqu'elle remonte à la fin du siècle précédent, est illustrée notamment par André Dacier, et encore par le Père Brumoy. Sélective, adaptatrice, elle veut respecter la norme de la clarté française et elle attribue volontiers aux anciens les usages et les manières de penser des modernes, qu'elle feint de croire universels. C'est elle que l'Abbé Goujet présente comme l'heureuse alliance de l'élégance et de la fidélité, bien préférable aux traductions poétiques de la Renaissance :

Si ces vieux traducteurs vous dégoûtent, contentez-vous de converser avec le père Brumoy qui nous a donné avec autant de fidélité que d'élégance, la traduction de quatre tragédies d'Euripide [...] Si une grande érudition est nécessaire pour corriger heureusement le texte des anciens auteurs, elle ne suffit pas toujours, et il faut que cette érudition se trouve pour l'ordinaire unie avec beaucoup de goût et de sagacité (Goujet, 17401756 , tome 4 , p. 163).

Mais toutes les traductions du siècle des Lumières ne sont pas de cette veine. Nous avons surtout voulu rendre toute sa place, dans la seconde moitié du siècle, à ce mouvement puissant, animé par des philologues mieux armés et plus méfiants à l'égard du goût classique, qui aboutit à promouvoir une nouvelle manière de traduire la tragédie grecque, que nous avons nommée la Traduction analytique en prose. 
Cependant, une troisième famille de traductions se dévèloppe en fin de siècle également, lointaine héritière de la démarche adaptatrice de la fin du XVI ${ }^{\mathrm{e}}$ siècle et de l'âge baroque, en réaction contre l'abandon de toute prétention poétique en traduction : c'est la Traduction-imitation.

\section{La Traduction-imitation au service de l'ambition littéraire}

Cette manière de traduire expérimente la voie envisagée en théorie par l'abbé Dubos et les artisans d'une conception impressionniste de l'œuvre d'art, tournée vers les effets qu'elle produit sur les sens. Elle procède par naturalisation du texte traduit selon les formes extérieures de la poésie de la langue cible ${ }^{2}$.

Dépendant de ses notes comme un invalide de ses béquilles, le texte des traductions analytiques doit sans cesse être rapporté à l'original à travers le fatras d'explications et de compléments que les notes accrochent à son cours. La forme que prennent les premières traductions analytiques au XVIII siècle a creusé un fossé entre la traduction et l'adaptation, fossé qui n'existait pas au $\mathrm{XVI}^{e}$ siècle - époque où la représentation des tragédies traduites en français était pratiquée $e^{3}$.

C'est contre ce manque d'ambition littéraire que réagissent les traducteurs-imitateurs. La Traduction-imitation peut " enchérir sur l'original ». Jean-François La Harpe l'énonce clairement, à l'occasion de la comparaison, dans son Cours de littérature, entre deux traductions d'un fragment de Cicéron. De la première version, qui s'apparente à la traduction analytique, il dit que " la prose explique ", tandis que de la deuxième version, en vers, due à Voltaire, le critique dit * voilà comme le poète traduit ou imite ". Le traducteur-imitateur a * mis une sorte d'ambition poétique à y ajouter de nouveaux coups de pinceaux. [...] Tous ces traits, et le dernier surtout qui est brillant, appartiennent à l'imitateur français. C'est

${ }^{2}$ Sur le concept de naturalisation appliqué à la traduetion, voir Tatilon, 1986, p. 26.

${ }^{3}$ Marie Delcourt précise à propos de la traduction de l'Antigone de Sophocle par Jean-Antoine de Baiff de 1572 que certains indices portent a croire que cette traduction a été représentée sur scène : * Qu'on suive les vers ou la mise en scène que le texte suggère, on a partout l'impression que Baif a traduit Antigone avec l'arrière-pensée de la faire jouer *, (Delcourt, 1925). 
une espèce de combat avec l'original; mais, pour l'entreprendre, il faut être bien sur de la trempe de ses armes ${ }^{4}$ (La Harpe, 1798, tome 1, p. 43-44). Le traducteur-imitateur espère surpasser l'auteur qu'il traduit, et pour cela, il compose un nouveau texte dont l'autonomie ne tient qu'à sa poétique propre.

Comme les traducteurs de cette époque se répondent, s'opposent parfois, échangent mutuellement leurs versions, en notes ou dans leurs préfaces, il est particulièrement intéressant de citer à notre tour deux traductions, l'une analytique, l'autre imitative, d'un même passage d'Hécube, ainsi que le commentaire que le traducteur analytique fait de la Traduction-imitation de son devancier.

La reine de Troie déchue supplie Ulysse de lui laisser sa fille, le dernier réconfort de ses vieux jours :

\section{EKABH}

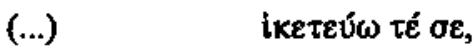

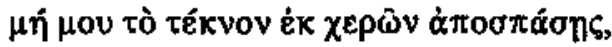

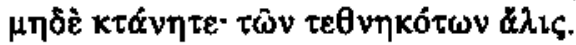

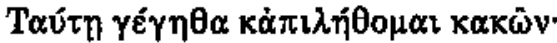

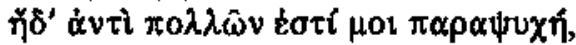

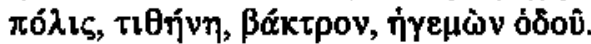

(Euripide, Hécube, 1973, v. 276-281).

Traduction française :

Hécube

[...] [et] je te supplie, n'arrache pas mon enfant de mes mains, ne la tuez pas ! Il y a eu assez de ceux qui ont péri. Par elle, je me réjouis, et j'oublie mes maux; elle est ma consolation contre tant de pertes, ma cité, ma nourrice, le bâton guide de mon chemin.

La Harpe, 1778 :

Hécube est à vos pieds, Hécube est reine, Hélas !

${ }^{4}$ Il s'agit de la traduction d'un extrait de Marius, imité de L'Iliade d'Homère. Les italiques sont de notre initiative. 
Hélas ! $n$ 'arrachez point ma fille de mes bras;

Ne versez point son sang : c'est assez de carnage :

Mes revers sont affreux : ma fille les soulage,

Console mes vieux ans, adoucit mes malheurs.

Prévost, 1785 :

Ulysse, je suis votre suppliante; $\delta$ Grecs n'arrachez point ma fille d'entre mes bras; $n$ 'altentez point à sa vie; c'est assez de ceux qui sont morts. Par elle, je suis heureuse, et j'oublie toutes mes infortunes; elle seule est pour moi tout ce que j'ai perdu : c'est ma consolation, ma patrie, ma nourrice, mon bâton de vieillesse, le guide de mes pas.

La traduction de La Harpe constitue un texte autonome. L'alexandrin régulier structure l'expression sans contrainte visible. Mais la forme versifiée semble ici déterminer les contenus. Les mots placés à la fin de chaque hémistiche se répondent en écho : * son sang " appelle * carnage ", " affreux * aboutit à * soulage ", et * mes vieux ans " renvoient à * mes malheurs ". Dans le premier vers, " Hécube est à vos pieds, Hécube est reine, Hélas ! ", le traducteur-imitateur se plaît à rappeler qu'Hécube, maintenant aux * pieds » d'Ulysse, est aussi « reine ». Ce contraste rehausse la requête qui va suivre. La répétition de l'interjection * hélas * en fin de vers et au début du vers suivant crée un effet de continuité qui ménage une association d'idées entre la situation de souveraine déchue et la sauvegarde d'une fille qui la soulage de cette déchéance.

La plupart de ces effets sont étrangers au texte d'Euripide, sans doute. Mais ne s'agit-il pas pour le traducteur d'un " combat avec l'original $n$ ? Il lui appartient de rivaliser d'invention pour trouver de nouvelles figures qui suscitent la même émotion que celles que le textesource pouvait éveiller dans l'âme des spectateurs de l'Antiquité. "Tout homme qui traduit en vers, dit encore La Harpe, prend la place de son modèle, et doit songer avant tout à plaire dans sa langue, comme l'auteur original plaisait dans la sienne. [...] De l'effet que fera sa version dépend l'opinion qu'auront de l'original ceux qui ne peuvent le connaitre autrement. [...] C'est donc à l'effet total de l'ensemble qu'il doit d'abord s'appliquer " (La Harpe, 1798). Pour susciter ces « effets ", le choix des moyens littéraires est libre, c'est-à-dire sans lien avec les mots, les sonorités, le rythme, les images de l'original. 
On ne doit pas s'étonner de la sévérité de Prévost, à l'égard de la version audacieusement libre de La Harpe qui, en transgressant cette loi de fidélité syntagmatique, bouscule les règles de bonne conduite du traducteur.

On sent qu'il serait injuste d'exiger qu'une traduction en vers conservât tous les traits de l'original sans les altérer. Ces images si naturelles et si touchantes qu'Hécube emploie pour exprimer ce que Polyxẻne est pour elle, M. de La Harpe n'a point osé les respecter, sans doute que la gene de la poésie ne lui en permettait pas l'usage. Il y a substitué, à l'exemple du Père Brumoy, une interjection qui, placée à la fin du vers, et répétée au commencement de celui qui suit, a de la grâce et un accent pathétique, mais qui peut-être dit moins à l'imagination (Prévost, 1785, * Avertissement ).

Pour Prévost, la poésie est une * gêne * pour le traducteur, une contrainte qui brime et entrave $\alpha$ l'imagination $n$. On pourrait lui opposer, presque mot pour mot, cette réflexion de La Harpe : "Une traduction en prose commence par anéantir l'art du poète, [...], cet heureux arrangement de mots, [...], ce concours de sons mesurés, [...] cette combinaison savante du mouvement et du rythme avec le sentiment et la pensée * (La Harpe, 1798). Mais cela nous éclaire-t-il vraiment sur les enjeux de deux méthodes si opposées?

La méthode théorisée et appliquée par Jean-François La Harpe ne résiste certes pas à la confrontation avec l'original. Il convient, pensons-nous, de replacer cette forme de traduction (la Traduction-imitation) dans le cadre d'une revendication de valeur littéraire, en réaction contre la traduction analytique. La démarche de La Harpe témoigne d'un début de renversement de perspective, non pas seulement vis-à-vis de la façon de traduire, mais par rapport à la propriété de l'œuvre d'art. Si les lecteurs đe traductions de tragédies grecques s'étaient contentés de la fidélité littérale des traductions analytiques, on en aurait conclu avec raison que l'œuvre originale est censée appartenir au domaine public, qu'elle constitue un matériau anonyme, un enchevêtrement de significations qu'il faut décoder, comme un ensemble de signes, ayant chacun son équivalent dans la langue cible. La démarche et l'exemple de La Harpe montrent que d'autres voix s'élèvent en son temps pour dire que l'œuvre appartient à son auteur, qu'elle est un cri jailli de ses entrailles, un message sorti de son cœur, une confidence secrète et intime, issue de l'expérience forcément singulière de l'homme, issue de son histoire individuelle, issue 
de sa confrontation empirique avec le langage, issue de sa douloureuse expérience de l'impuissance des « mots de la tribu a à se dire. Cette voix ne risque-t-elle pas de périr, si on la soumet à une opération de décodage-encodage, régie par la combinaison d'une grille syntaxique et d'un système de correspondances lexicales imparfaites?

Traduire, est-ce conserver une structure syntaxique, et revêtir les mots d'une langue, un à un, par les mots d'une autre langue? Ce pourrait être cela, si les mots étaient interchangeables, si les langues étaient des magasins de signification, des listes d'étiquettes. Charles Péguy a dénoncé ce piège :

Vous me dites que Oiði $\pi \pi$ ous n'est pas Edipe. Croyez-vous que túpavvos fasse roi? Croyez-vous que iepeús fasse prêtre, et même sacrificateur? au sens que ces deux mots éveillent dans un esprit, dans une âme moderne, en admettant que le deuxième éveille un sens dans un esprit ou dans une ame moderne. Croyez-vous que $\beta \omega \mu$ oi soient autels, au sens que nous entendons autels? Et même croyez-vous que tékva soient enfants, et ne savez-vous pas qu'il y a aussi des distances autant irrémissibles entre ce que tếvo éveillait dans les échos de l'âme antique et ce que enfants éveille dans les autres échos de l'âme moderne. Tékva

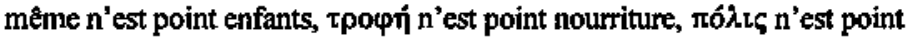
seulement ville ni cité; puisque c'est nommément une cité grecque; rien n'est rien; rien ne se refait parfaitement, rien ne se recommence, rien ne se reproduit exactement, rien d'ancien n'est en même temps nouveau, rien de nouveau n'est en même temps ancien; de tout à tout il subsiste éternellement des distances irrémissibles; et c'est pour cela que toute opération de traduction est essentiellement, irrévocablement, irrémissiblement une opération miséreuse, une opération misérable et vaine, une opération condamné (Péguy, 1905, p. 35).

Or la traduction n'a pas à être fidèle à des lexiques, ni même à des langues, mais seulement à des textes, qui sont le vouloir-dire d'un auteur, et non le vouloir-dire d'une langue (Pergnier, 1993, p. 24). La Harpe pose ici une question essentielle : peut-on traduire sans interpréter? sans dire à nouveau « je ? Ne faut-il pas jouer cette partition avec le secours de sa propre expérience du monde et des mots, pour faire entendre une voix qui soit à la ressemblance du modèle? La Harpe, professeur de littérature, rend hommage aux formes poétiques de son époque en même temps qu'il valorise une certaine idée de la création artistique, émanation d'une conscience singulière qui structure le monde tel qu'elle le sent. 
D'autres théoriciens du début du $\mathrm{XIX}^{\complement}$ siècle reprendront les idées de La Harpe. Dureau-Delamalle estime en 1805 que pour surmonter les différences culturelles qu'il rend responsables de la non-concordance des lexiques d'une langue à l'autre, il faut se laisser pénétrer du style de l'auteur pour le retrouver en soi-mème : Ne pouvant plus être guidé par la phrase ou grecque ou latine dont les mots [...] ne produiraient plus les mêmes effets, [...] il [le traducteur] est obligé de se créer une diction nouvelle, de saisir de nouveaux rapports, de former de nouvelles combinaisons. [...] [le traducteur devra donc] pénétrer comme lui [l'auteur] dans toute la profondeur de la pensée, pour retrouver toutes les qualités de son style " (Dureau-Delamalle, 1805). Le mode de traduction proposé par La Harpe est la première critique fondamentale adressée à la Traduction analytique en prose. L'observation des traductions d'Hécube au $\mathrm{XIX}^{\bullet}$ et au $\mathrm{XX}^{\mathrm{e}}$ siècle montre que d'autres méthodes de traduction prendront acte des arguments fondamentaux développés à la fin du XVII' siècle pour dénoncer l'imposture que représente, pour traduire les poètes, une fidélité restreinte a l'articulation grammaticale des éléments lexicaux. Mais elles resteront minoritaires. La fidélité, syntaxique d'abord, lexicale plus tard, l'emportera bientôt dans ce débat, tout au moins en ce qui concerne la traduction française des tragédies grecques, jusqu'à une date récente.

La principale conséquence des questions débattues et des options retenues durant cette période fondamentale est certainement la polarisation du débat theorique. Il faut depuis lors choisir entre ces deux voies, la Traduction analytique en prose et la Traduction-imitation, ou, pour dire les choses plus simplement, entre les laides fidèles et les belles infidèles. N'y aura-t-il plus jamais en France de recherche en matière de traduction poétique des tragédies grecques, visant à produire de belles fidèles? Les premiers traducteurs français de tragédies grecques, au milieu du XVI ${ }^{e}$ siècle, avaient marché sur cet étroit chemin. Il s'agissait alors de découvrir, de connaître, de pénétrer un mystère, et la poésie n'était point ennemie de l'étude. Ce n'est pas l'érudition que le classicisme français a tuée, c'est cette proximité du savant et du poète, qui fut, l'espace d'un demi-siècle, entre 1540 et 1570, l'âge d'or de la traduction poétique des tragiques en France.

Aujourd'hui, la quête du sens, l'etude des grands mythes qui sont des instruments de la pensée, les metteurs en scène du théâtre présent, qui demandent des traductions nouvelles, appellent un autre apprentissage des 
ceuvres de l'Antiquité que la dissection grammaticale, et une autre communication de la tragédie grecque aux lecteurs français que la prose glacée des traductions savantes.

\section{Institut Universitaire de Formation des Maîtres de l'académie de Corse}

\section{Références}

ABLANCOURT, Nicolas Perrot d' (1637). L'Octavius de Minucius Felix, Paris, J. Camusat.

BAlF, Jean-Antoine ([1572] 1881-1890). Sophocle, Antigone, dans CEures en rime de Ian Antoine de Baif, secrétaire de la chambre du roy, avec une notice biographique et des notes, par Marty-Laveaux Ch., Paris, A. Lemerre.

BALLARD, Michel, D'HULST, Lieven (1996). La traduction en France à l'age classique, Villeneuve d'Asq, Presses Universitaires du Septentrion.

BELLANGER, Justin (1903). Histoire de la traduction en France (auteurs grecs et latins), Paris, E. Thorin.

BERTRAND, Louis ([1897] 1968). La fin du classicisme et le retour à l'antique dans la seconde moitié du XVIIF siècle et les premières années du $X I X^{\circ}$ siècle, en France, Paris, Genève, Slatkine Reprints.

BIET, Christian (1982). « Digressions, infléchissements, ajouts dans les traductions d'Edipe-Roi au XVIII" siècle ", Questions de sens : Homère, Eschyle, Sophocle, Aristote, Virgile, Apulée, Clément, Études de Littérature Ancienne, tome 2, Paris, Presses de l'École Normale Supérieure [P.E.N.S.], pp. 123-143.

BLIGNIÈRES, Auguste de ([1851] 1968). Essai sur Amyot et les traducteurs français du XVF siècle, Paris, Genève, Slatkine Reprints.

BOILEAU, Nicolas ([1674] 1966). Art poétique, auvres complètes, Paris, Adam-Escal, Gallimard. 
BRUMOY Pierre (1730). [trad.], Théâtre des Grecs par le R. P. Brumoy, Paris, Rollin père et fils, J.-B. Coignard.

COLLERET, Guillaume ([1636] 1658). Art poëtique... Avec un Discours de l'Eloquence, et de l'Imitation des Anciens. Un autre discours contre la Traduction..., Paris, A. de Sommaville et L. Chamhoudry.

DACIER, André (1692). L'GEdipe et l'Electre de Sophocle, tragédies grecques traduites en français, avec des remarques, par André Dacier, Paris, Cl. Barbin.

DELCOURT, Marie (1925). Etude sur les traductions des Tragiques Grecs et Latins en France depuis la Renaissance, Bruxelles, Lamertin.

DESFONTAINES, Pierre François Guyot, Abbé - (1757). « Discours sur la traduction des poètes ", $L$ 'esprit de l'Abbé Desfontaines ou réflexions sur les différents genres de science et de littérature, Paris, 4 vol., tome 1, pp. 271-306.

D'HULST, Lieven (1990). Cent ans de théorie française de la traduction : de Battexx à Littré, 1748-1847, Lille, Presses Universitaires de Lille.

DIDEROT, Denis ([1751] 1875-1877). Lettre star les sourds et muets, dans CEuvres complètes, Assézat-Toumeux, [éd.], Paris Garnier Frères, vol. I.

DIDEROT, Denis ([1762] 1875-1877). Réflexions sur Térence, dans Euvres complètes, Assézat-Toumeux, [éd.], Paris Garnier Frères, vol. V.

DUBOS, Abbé Jean-Baptiste, ([1719] 1993). Réflexions critiques sur la poésie et sur la peinture, Désirat, Dominique, [éd. et préface], Paris, École nationale supérieure des beaux-arts.

DUREAU-DELAMALE, Jean-Baptiste Joseph René (1805). Discours prononcés dans la séance publique temue par la classe de la langue et de la littérature françaises de l'Institut National, le 11 floréal an XIII pour la réception de M. Dureau-Delamalle, [Bibliothèque Nationale de France $4^{\circ}$ Z 266]. 
DU RYER, Pierre (1639). Les Philippiques de Ciceron. Premiere Partie. Paris, A. de Sommaville.

ESCHYLE (1770). Le Franc de Pompignan, Jean-Jacques, [trad.], Tragédies d'Eschyle traduites en français, Paris.

ESTIENNE, Henri (1611). Lexicon Gracolatinum, seu epitome thesauri Graces lingua ab Henrico Stephano constructi..., Apud Petrum Baldinum.

EURIPIDE (1544). Bochetel, Guillaume, [trad.], La tragedie d'Euripide nommée Hecuba, traduicte de Grec en rhythme Françoise, dediee au Roy, Paris, Robert Estienne imprimeur du Roy.

EURIPIDE (1783). Belin de Ballu [trad.], Hécube, première tragédie d'Euripide traduite en français avec des remarques, Paris.

EURIPIDE (s. d.). [traducteur anonyme], Hécube, [fin XVIII' siècle], traduction manuscrite française en prose, Mélanges de philologie et de littérature grecque et latine, [cote B.N.F. : MS français 9299(2)].

EURIPIDE (1829). Philippe Lebas, [trad.], Hécube, par detax traductions, l'une littérale et interlinéaire, avec la construction du grec dans l'ordre naturel des idées, Paris.

EURIPIDE (1836). V. H. Chappuyzi, [trad.], Professeur au collège Royal de Saint-Louis, Hécube Tragédie d'Euripide, Texte pur et traduction correcte, avec analyse, notes françaises et version interlinéaire, Paris.

EURIPIDE (1901). Hécube, C. Leprévost, [trad.], Paris, Hachette, * Les auteurs grecs par deux traductions françaises, l'une littérale et juxtalinéaire présentant le mot à mot français en regard des mots grecs correspondants, l'autre correcte et précédée du texte grec ".

EURIPIDE ([1927] 1973). Théâtre d'Euripide, tome 2, Hippolyte, Andromaque, Hécube, texte et traduction, Méridier, Louis, [éd. et trad.], Paris, Les Belles Lettres.

FONTENELLE, Bernard Le Bovier de ([1688] 1993). Digression sur les Anciens et les Modernes, Euvres completes, Paris, Fayard. 
GARNIER, Bruno (1998). « La Traduction dans l'enseignement des langues anciennes : les mots contre le sens? ", DELISLE, Jean et LEE-JAHNKE, Hannelore [dir.], Enseignement de la traduction et traduction dans l'enseignement, Préface de Maurice Pergnier, Ottawa / Arras, Les Presses de l'Université d'Ottawa / Artois Presses Université.

GARNIER, Bruno (1998). Pour une poétique de la traduction : L'Hécube d'Euripide en France de la Renaissance à l'age classique, Paris, L'Harmattan.

GIRY, Louis (1636). Apologetique, ou Defense des Chrestiens contre les accusations des Gentils, traduit en François du Latin de Tertullien, Paris, J. Camusat.

GOUJET, Claude-Pierre, Abbé - (1740-1756). Bibliothèque Françoise ou Histoire de la littérature françoise, Paris, Mariette, Guérin, Lemercier et Delatour, 18 vol.

HOBIER, Ithier (1639). Tacite, de la vie d'Agricola son beau-pere, Paris, J. Camusat.

HORN MONVAL, Madeleine (1958). Repertoire bibliographique des traductions et adaptations françaises du théâtre étranger, Paris, CNRS.

HUET, Pierre Daniel (1661). De interpretatione libri duo : quorum prior est De optimo genere interpretandi; alter, De claris Interpretibus, Parisiis, apud S. Cramoisy.

LA HARPE, Jean-François (1778). [Traduction d'extraits d'Hécube], Essai sur le théatre grec, Eavres de Monsieur de La Harpe, Paris, tome 1.

LA HARPE, Jean-François ([1798] 1815). Lycée ou Cours de Littérature ancienne et moderne, Nouvelle édition, Paris, E. Ledoux et Tenré, tome 1.

LA ROCHEFOUCAULD ([1665] 1954). Maximes et réflexions morales, Paris, Larousse.

LA ROVIÈRE, Pierre de (1614), Poetae Graci veteres tragici, lyrici, comici, epigrammatarii, additis fragmentis exprobatis authoribus collectis, 
nunc primum Grace et Latine in unum redacti corpus, Colonia Allobrogum.

MARCELLO, Benedetto ([1720] 1993). Le théâtre à la mode au XVHIe siècle, Traduit de l'italien « Il teatro alla moda * de Benedetto Marcello, par Jacques Drillon, Arles, Bernard Coutaz.

MIRAMBEL, André (1962). La France devant l'hellénisme, Paris, Les Belles Lettres.

MONTFAUCON, Bernard de ([1719] 1757). Antiquitates graecae et romanae a viro [...] Bernardo de Montfaucon [...] explanatae et schematibus illustratae, nunc autem ad commodiorem studiosae inventutis usum in compendium redactae, et figuris aeneis forma minori exornatae a Johanne Jacobo S. [...], Norimgergae, Impensis Georgii Lichtenstegeri.

MOUNIN, Georges (1994). Les belles infidèles, Ballard, Michel, [préface], D’Hulst, Lieven, [préface], Lille, Presses Universitaires de Lille.

PARFAIT, Les Frères - (1745-1749). Histoire du théâtre françois, depuis son origine jusqu'à présent avec la vie des plus célebres Poëtes dramatiques, un catalogue exact de leurs Pieces, et des notes historiques et critiques, Paris, 12 vol.

PEGUY, Charles ([1905] 1988), " Les Suppliants Parallèles », Les Cahiers de la Quinzaine, VII, vii, [17/12/1905], dans Charles Péguy, Euvres en prose complètes, tome II, Paris, Gallimard, la Pléiade.

PERGNIER, Maurice (1993). Les Fondements socio-linguistiques de la traduction, $3^{e}$ édition remaniée, Presses Universitaires de Lille.

PETIT DE JULLEVILLE, L. (1885). Histoire du thếtre en France, Paris.

PRÉVOST (1785). Le Théâtre des Grecs par le Père Brumoy, Nouvelle édition enrichie de gravures, augmentée, traduction entière des pièces grecques par MM de Rachefort et du Theil, pour les trois premiers volumes et par M. Prévost pour les dix autres, Paris, Cussac [pour Sophocle et Eschyle] / Brothier [pour les dix volumes de traduction d'Euripide par Prévost. 
RACINE ([1674] 1994). Iphigénie, « Préface », Paris, Classiques Hachette.

ROCHEFORT, Guillaume Dubois de (1793). Hécube, tragédie en trois actes et en vers, imitée du grec d'Euripide, lue et reçue à la Comédie Française le 4 août 1792 , précédée d'un essai de traduction de quelques scènes du théâtre grec, Paris, Citoyen Cailleau.

ROMILLY, Jacqueline de (1979). * La traduzione dei testi teatrali antichi in Francese (1700-1900) ", Dioniso : rivista di studi sul teatro antico, Siracusa.

ROMILLY, Jacqueline de ([1970] 1992). La tragédie grecque, Paris, Presses Universitaires de France, $4^{\complement}$ éd.

SAÏD, Suzanne (1978). La faute tragique, Paris, Maspéro.

SOPHOCLE ([1958] 1985). Théâtre de Sophocle, texte et traduction, tome II, Ajax, Gdipe roi, Electre, Dain, Alphonse, [éd.], et Mazon, Paul, [trad.], Irigoin, Jean [éd.], Paris, Les Belles Lettres.

TATILON, Claude (1986). Traduire: Pour une pédagogie de la traduction, préface de Georges Mounin, Toronto, Editions du Groupe de Recherche en Études Francophones, Coll. «Traduire, écrire, lirew.

VAN BRAGT, Katrin, LAMBERT, José, D'HULST, Lieven (1995). Bibliographie des traductions françaises (1810-1840) : répertoire par disciplines, Louvain, Presses Universitaires de Louvain.

VAULTIER, Marie Claude Frédéric Etienne (1812). De la Traduction, thèse de littérature ancienne et moderne, Université impériale, Académie de Paris, Faculté des Lettres, [Bibliothèque Nationale de France $4^{\circ} \mathrm{X}$ piècel 17].

ZUBER, Roger ([1968] 1995). Les "belles infideles " et la formation du gout classique : Perrot d'Ablancourt et Guez de Balzac, postface d'Emmanuel Bury, Paris, Albin Michel, édition revue et augmentée.

RÉSUMÉ : La traduction de la tragédie grecque en France. Le tournant décisif de la période 1660-1780 — La traduction de la tragédie 
grecque en France, dont l'histoire avait commencé à la Renaissance, connaît une longue interruption au XVII' siècle, qui correspond à la naissance et au développement de la tragédie classique française. C'est sur la période suivante, durant laquelle la traduction de la tragédie grecque a repris en France, que nous voulons mettre l'accent, parce que les façons de traduire qui ont prévalu à l'issue de ce nouvel élan ont fortement marqué l'histoire ultérieure du genre et elles ont continué de prévaloir, en ce qui concerne la tragédie grecque, jusqu'à une date récente. C'est au lendemain des vifs débats sur la manière de traduire les poètes et à la suite des progrès de l'érudition et de la philologie que s'est développée en France la Traduction analytique en prose, fruit d'un intérêt nouveau pour l'Antiquité et d'un code de bonne conduite implicite des traducteurs. En réaction contre la prose glacée des traductions savantes, à l'autre extrémité du spectre interprétatif, a pris naissance la Traduction imitation, audacieusement libre, lointaine héritière des traductions de l'âge baroque. Depuis lors, le débat est polarisé entre ces deux options, alors que les conditions semblent aujourd'hui réunies pour que soit restaurée cette proximité du savant et du poète qui avait fait l'âge d'or de la traduction poétique à la Renaissance.

\begin{abstract}
The Turning Point in the Translation of Greek Tragedy in France - The translation of Greek Tragedy in France, which began during the Renaissance, was internupted by the development of the French Classical tragedy during the entire $17^{\text {th }}$ century. Following this interruption, the French translation of Greek tragedies resumed and greatly influenced the genre. With regard to Greek tragedy, the methods invoked continued to prevail until recently. Following the heated debate over the methodology of translating the classics, the Analytic Prose school was developed. This method, based on an implicit translator's code of conduct, was bolstered by progress in erudition and philology due to increased interest in the Antiquity. However, the Imitating Translation school soon arose in reaction to the stilted prose of scholarly translation. This audaciously free method, diametrically opposed to that of the Analytic Prose school, was a distant heir to Baroque-era translations. Since that time, the debate has been polarized between these two options, although the time may be ripe for the condition which marked France's golden age of poetic translation during the Renaissance.
\end{abstract}

Bruno Garnier : Institut Universitaire de Formation des Maîtres de l'Académie de Corse, 2, rue de l'Eglise, B.P. 86, 20250 Corte, France. Courriel : brunopat@chub-internet.fr 